\title{
Propuesta metodológica para la investigación de la comunicación en las organizaciones
}

\section{Proposta metodológica para pesquisa em comunicação em organizações Methodological proposal for communication research in organizations}

\section{Manuela Matus Verdugo}

Candidata a doctora en Planeación Estratégica y Dirección de Tecnología por la Universidad Popular Autónoma de Puebla, México

- Maestra en Comunicación por la Universidad Nacional Autónoma de México

- $\quad$ Profesora investigadora de la Universidad de Sonora

- Coordinadora del equipo diseñador de la Maestría en Comunicación Estratégica

- Coordinadora del Eje Especializante de Comunicación Organizacional en la Universidad de Sonora

- Fue Coordinadora de Prácticas Profesionales en Ciencias de la Comunicación

- $\quad$ Autora de los capítulos del libro "Prácticas profesionales de comunicación en las organizaciones" (Las ciencias informáticas. Generación del conocimiento en entornos educativos: un enfoque multidisciplinario. México, DF: Ediciones ILCSA, 2017) y "Netnografía de Prácticas Profesionales de Comunicación Organizacional en México" (Educación y universidad ante el horizonte 2020: inclusión y cultura colaborativa entre empresa y sociedad. México, DF: Qartuppi, 2017. v.3)

- E-mail: manuela.matus@unison.mx

\section{Jorge Alfredo Estupiñán Munguía}

Pasante de la Maestría en Comunicación por la Universidad Nacional Autónoma de México

- Profesor investigador de la Universidad de Sonora

- Socio fundador de Estupiñán \&Tena, Consultores en Comunicación y Desarrollo Organizacional

- Diseñador y Coordinador del Diplomado en Comunicación Organizacional y Relaciones Públicas

- Fue director de Extensión Universitaria de la Universidad de Sonora, Secretario Académico de la División de Ciencias Sociales y Vicepresidente del Consejo Nacional para la Enseñanza y la Investigación de las Ciencias de la Comunicación (Coneicc)

- $\quad$ Autor del capítulo de libro "Ambientes organizacionales de aprendizaje y educación continua" (Educación continua y a distancia en las universidades de México, 2018)

- E-mail:jae@sociales.uson.mx

\section{Ma Elena Zermeño-Espinosa}

- Doctora en Ciencias de la Comunicación Social por la Universidad de La Habana

- $\quad$ Profesora-investigadora de la Universidad Autónoma de Baja California

- Miembro del Cuerpo Académico Procesos de Comunicación en Organizaciones e Instituciones Sociales (Procois)

- Coordinadora de la Unidad de Servicios Integrales en Comunicación (Usic)

- Autora del libro Estrategias Comunitarias de Comunicación para la Salud (Eccos) en una comunidad fronteriza del norte de México (Guadalajara: Ediciones de la Noche, 2018); autora del capítulo de libro "Experiencias organizacionales para el trabajo comunitario rural en pro de su salud" (Estudios empresariales: retos y oportunidades para México. Heredia: Izcandé, 2018)

- E-mail: maelena@uabc.edu.mx 


\section{Resumen}

El propósito del trabajo es presentar una propuesta metodológica para investigar la comunicación en las organizaciones, considerando la dimensión praxeológica del ámbito educativo, así como la profesional. Se identifican tres actores clave: el practicante profesional, es decir, el estudiante que se acerca al mundo profesional como parte de su formación académica; el profesional en Comunicación, quien soluciona necesidades de comunicación en organizaciones del sector público, social y privado; y las organizaciones representando, para el primero, la unidad receptora y, para el segundo, el mercado laboral.

\section{PALABRAS CLAVE: PROPUESTA METODOLÓGICA•DIMENSIÓN PRAXEOLÓGICA•COMUNICACIÓN ORGANIZACIONAL.}

\section{Resumo}

O objetivo do trabalho é apresentar uma proposta metodológica para investigar a comunicação nas organizações, considerando a dimensão praxeológica do campo educacional, bem como o profissional. Três atores-chave são identificados: o profissional, ou seja, o aluno que aborda o mundo profissional como parte de sua formação acadêmica; o profissional de Comunicação, que resolve as necessidades de comunicação em organizações públicas, sociais e do setor privado; e organizações representando, para o primeiro, a unidade receptora e, para o segundo, o mercado de trabalho.

\section{PALAVRAS-CHAVE: PROPOSTA METODOLÓGICA • DIMENSÃO PRAXEOLÓGICA•COMUNICAÇÃO ORGANIZACIONAL.}

\section{Abstract}

This article aims to present a methodological proposal to investigate communication in organizations, considering the praxeological dimension of the educational environment, as well as the professional. Three key actors are identified: the student, who approaches the professional world as part of their academic preparation; the communication professional, who solves communication needs in public, social and private sector organizations; and the organizations, representing, for the first mentioned, the receiving unit, and, for the second one, the labor market. 


\section{INTRODUCCIÓN}

a propuesta metodológica tiene como origen la línea de investigación Campo Profesional de la Comunicación en las Organizaciones, registrada en la Academia de Comunicación Estratégica y Globalización de la Universidad de Sonora, México; la vinculación con la Asociación Mexicana de Investigadores y Profesionales de Comunicación Organizacional Ampico y los trabajos de la reunión de redes académicas que se realizaron en el marco del Encuentro Coneicc Noroeste los días 16 y 17 de marzo de 2018, en Hermosillo, Sonora, México y cuya sede fue la Universidad de Sonora a través de la licenciatura en Ciencias de la Comunicación. Un evento cuyo objetivo fue propiciar el diálogo, reflexión e intercambio de experiencias para lograr un acercamiento epistemológico y profesional a las tendencias críticas y a los nuevos retos existentes en el campo de la Comunicación en México.

Dicho encuentro permitió fortalecer el trabajo de la red de investigadores, si bien las interacciones habían sido virtuales a través del Seminario Mexicano de Comunicación Organizacional Semco que realiza la Amipco a través de Internet, por primera ocasión se realizó una reunión de manera presencial, dada la gestión del maestro Jorge Alfredo Estupiñán Munguía, Coordinador del Eje Especializante de Comunicación Organizacional de la licenciatura en Ciencias de la Comunicación de la Universidad de Sonora y la maestra Griselda Guillén Ojeda, presidenta de dicha Asociación, quien participó en el Encuentro Coneicc con una agenda amplia que incluyó la presentación del libro La Comunicación para las Organizaciones en México. Evolución, teoría y práctica; participación como ponente en mesa de expertos en Comunicación; reunión con la red de investigadores, así como instructora de taller. El encuentro evidenció el trabajo colaborativo como estrategia para el fortalecimiento de la Comunicación Organizacional y puso sobre la mesa de discusión la práctica profesional de la comunicación en las organizaciones.

Desde esta perspectiva y de acuerdo al primer Modelo heurístico de las estructuras del campo académico de la comunicación de Fuentes Navarro (1988) configurado por los subcampos Científico, Educativo y Profesional, se retoma el último para la descripción del presente trabajo, porque en este se concentran las prácticas de aplicación del conocimiento, una preocupación para Fuentes Navarro (2015) pues considera que no se tiene idea de qué sucede en ese vértice de la profesión, ya que su objeto de indagación se ha centrado en la investigación académica como práctica relacionada con el desarrollo teórico y la relación que tiene ese subcampo científico con el subcampo educativo (Figura 1). 
Figura 1: Estructura del campo académico de la comunicación

\section{Estructura del campo académico de la comunicación (Primer modelo heurístico)}

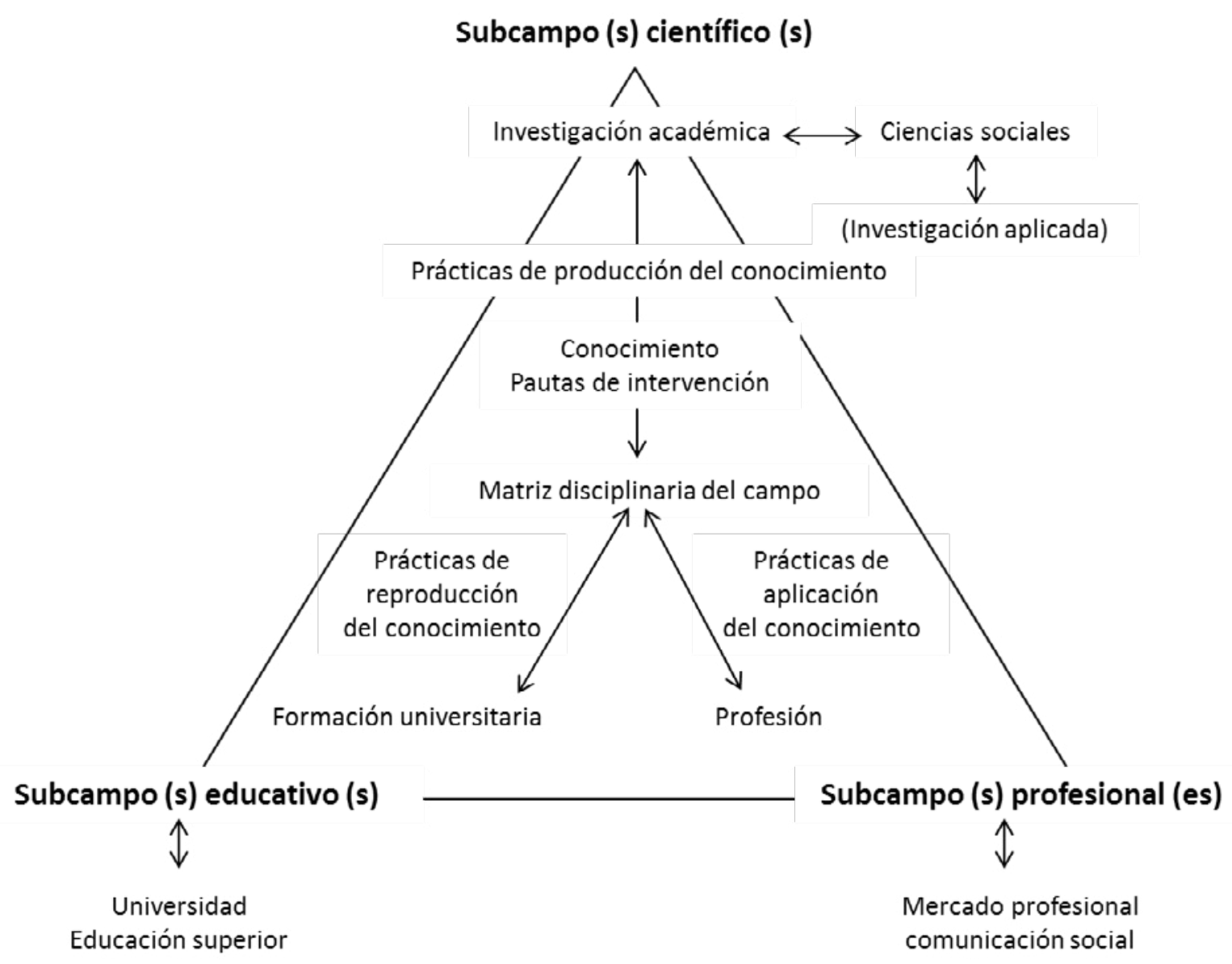

Fuente: Fuentes Navarro (1998).

En coincidencia con lo que plantea Fuentes Navarro, se observa que la doctora Consuelo Vásquez, de la Universidad de Québec à Montreal, Canadá, trabaja sobre la perspectiva constitutiva de la Comunicación Organizacional. Según la autora, en entrevista que le hiciera la Amipco (Consuelo..., 2017), dicha perspectiva teórica propone y pone en el centro de la organización y de los procesos organizacionales a la comunicación, pues tiene como premisa básica que las organizaciones están constituidas por prácticas comunicacionales referidas a textos, conversaciones, encuentros, disputas, conflictos; todo lo que pasa a través del habla, de la escucha, de los cuerpos, del lenguaje no verbal y de los espacios. A pregunta expresa del entrevistador sobre cómo podría Latinoamérica aportar a este trabajo de investigación, la autora aseveró que desde la dimensión aplicada para llevar esos conceptos a la práctica [Subcampo profesional], reconoce que es un trabajo que no han hecho como, por ejemplo, intervenir para ayudar a la micro, pequeña y mediana empresa (Mipymes) a desarrollar su competitividad y reconocer espacios de diálogo, entre otros.

Desde el subcampo educativo, las Instituciones de Educación Superior, IES, han incorporado en el currículo las prácticas profesionales, una recomendación que la Unesco (1998) hiciera desde 1998 a fin de reforzar y renovar los vínculos entre la enseñanza superior, el mundo del trabajo y otros sectores de la sociedad. Con esta vinculación se ha logrado una serie de acciones positivas para las instituciones educativas, estudiantes y organizaciones, como la firma de 923 convenios concentrados de los cuales 696 son nacionales y 227 internacionales. En materia de prácticas profesionales, por ejemplo, la Universidad de Sonora, a través de la Dirección de Vinculación y Difusión, registra, hasta la fecha, 46 convenios con diversas organizaciones de las cuales seis están ubicadas en el sector público, cinco en el sector social y 35 en el sector privado. En materia de prácticas profesionales y servicio social, registra 46 convenios, 21 son de organizaciones del sector público y 25 en el sector social. Es decir, los practicantes tienen un total de 92 unidades receptoras como opción para realizar sus prácticas profesionales PP (Tabla 1). 
Tabla 1: Convenios de colaboración para realizar prácticas profesionales

\begin{tabular}{c|c}
\hline \multicolumn{2}{c}{ Convenios en materia de prácticas profesionales } \\
\hline Sector & Total \\
\hline Sector público & 6 \\
\hline Sector social & 5 \\
\hline Sector privado & 35 \\
\hline Total & 46 \\
\hline \hline Convenios en materia de prácticas profesionales y Servicio social \\
\hline Sector & Total \\
\hline Sector público & 21 \\
\hline Sector social & 25 \\
\hline Total & 46 \\
\hline
\end{tabular}

Fuente: Elaborada por los autores con información de la Dirección de Vinculación y Difusión de la Universidad de Sonora (2018).

Además de la firma de convenios que formaliza las prácticas profesionales (PP), académicos y estudiantes de la Licenciatura en Ciencias de la Comunicación, han gestionado el vínculo con diversas organizaciones para acercarse al mundo del trabajo, formalizando la relación a través de formatos que son signados por tres actores clave: el académico, el estudiante y el representante de la organización y que, de acuerdo a la normatividad (Reglamento General de Prácticas Profesionales, 2017), se identifican como Tutor de Prácticas Profesionales, Practicante y Unidad Receptora, respectivamente. Una tríada que, desde el ámbito educativo, podría posibilitar no solo el acercamiento del estudiante al campo de su profesión, sino también realizar estudios sobre la importancia de la comunicación para las organizaciones, es decir, la dimensión praxeológica que los investigadores reconocen que es necesario investigar.

Sin embargo, para robustecer el estudio se considera no solo la participación de los practicantes desde al ámbito educativo, sino también a los egresados en el ámbito profesional de la comunicación, para que compartan sus experiencias de acuerdo a su trayectoria en las organizaciones donde han laborado.

En estos ámbitos, educativo y profesional, aparecen las organizaciones por lo que se convierten en actores clave para estudiar la comunicación con base en el quehacer especializado en una subdisciplina de las Ciencias de la Comunicación: la Comunicación Organizacional, por lo que practicantes, profesionales de la comunicación y organizaciones, son actores clave en la investigación, pues a través de ellos se podría conocer el contexto de la comunicación en las organizaciones, así como el quehacer y desarrollo profesional del comunicador. Al reconocer la viabilidad de la investigación, dada la vinculación con dichos actores, se presenta una propuesta metodológica con la que se pretende investigar la dimensión praxeológica de la comunicación en las organizaciones.

\section{PROPUESTA METODOLÓGICA PARA LA INVESTIGACIÓN DE LA COMUNICACIÓN EN LAS ORGANIZACIONES}

Para estudiar la comunicación en las organizaciones, se propone en principio el acercamiento al subcampo profesional de la Comunicación Organizacional, desde dos ámbitos que permitirán conocer el ejercicio profesional del comunicador en las 
organizaciones: el educativo y el profesional. En ambos, aparecen tres actores clave que se relacionan entre sí, practicantes, organizaciones y profesionales de la comunicación.

La vinculación entre ellos no es una acción novedosa, pues las IES que siguen las recomendaciones para acercar a los estudiantes al mundo del trabajo institucionalizan las prácticas profesionales como asignaturas obligatorias en sus programas educativos y las consideran, incluso, como una modalidad de titulación. Las intervenciones de los practicantes, como lo señalan Matus Verdugo, Valenzuela y Estupiñán Munguía (2017), han sido en organizaciones públicas, sociales y privadas, en diversos sectores económicos, por lo que las intervenciones del comunicador, van más allá de los medios de comunicación y de las instituciones.

La propuesta trata de aprovechar estratégicamente los vínculos que establecen los actores desde los dos ámbitos, para la generación de conocimiento desde el subcampo profesional, como se describe en la Figura 2:

Figura 2: Acercamiento al subcampo profesional de la comunicación en las organizaciones

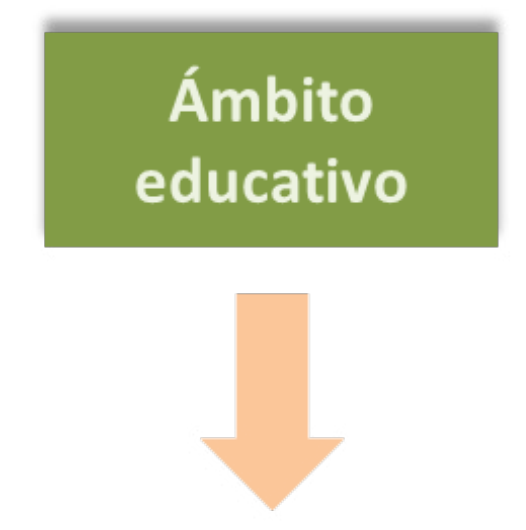

Practicantes

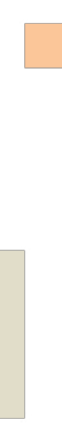

Organizaciones

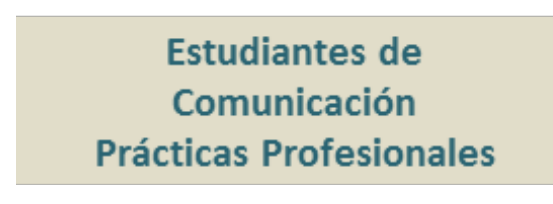

Fuente: elaborada por los autores.

\author{
Acercamiento al Subcampo \\ profesional de la Comunicación \\ en las Organizaciones
}

Ámbito

profesional

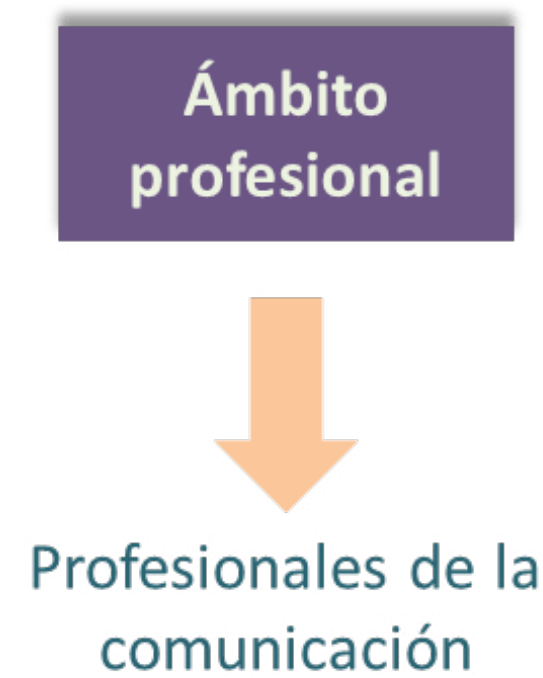

Egresados de Comunicación Responsables de la comunicación organizacional

\section{ÁMBITO EDUCATIVO}

La estrategia se estructura desde el subcampo académico y parte de una serie de preguntas que deben ser consideradas para el diseño, validación y aplicación de instrumentos tanto cuantitativos como cualitativos, como: ¿Qué factores intervienen para que, desde la Comunicación, sea posible la exploración de las organizaciones? ¿De quién depende la Comunicación en el organigrama organizacional? ¿Qué relación tiene la teoría con su práctica profesional? ¿Qué formación académica tienen los responsables de la comunicación en las organizaciones? ¿En qué sector se invierte más en comunicación? ¿Cuál es la misión y visión? ¿Cuáles son sus objetivos? ¿Cuáles son los recursos tecnológicos que tienen para la información y comunicación? ¿Quiénes son los responsables de la comunicación?

Los principales actores en este ámbito son los estudiantes y las organizaciones, los primeros reconocidos también como practicantes, y los segundos como unidades receptoras, empleadores y mercado de trabajo. 


\section{ANO 16 • NÚMERO 30 • 1은. 2019 • ORGANICOM}

\section{CÓMO IMPLEMENTAR LA ESTRATEGIA}

1) La práctica profesional que realizan los universitarios en las organizaciones supone, para su evidencia, el desarrollo de un proyecto en el que tendrá que describir el contexto de la organización. Para este caso, un primer acercamiento a la información contextual será la aplicación de un instrumento que dé respuesta a los cuestionamientos antes señalados.

2) Desde el subcampo académico, se proporcionará el instrumento. Para ello, se diseñará una base de datos que facilite la captura de información de las organizaciones presentadas en equipo o de manera individual por los practicantes de comunicación.

3) El alumno tendrá control de su información; sin embargo, se le aclarará desde un principio, que la aplicación de los instrumentos proporcionados y la información obtenida serán considerados en un estudio amplio sobre la comunicación en las organizaciones.

4) Dado que la práctica profesional es supervisada, el mismo académico podrá aplicar la primera parte del instrumento, aun así, el alumno utilizará la información para que la incluya en la descripción del contexto. Es necesario advertir a la unidad receptora sobre el primer instrumento que se aplicará para describir el contexto de la organización en materia de comunicación.

5) El instrumento se conformará en tres apartados: i) Contextual, que se construye con las preguntas planteadas al respecto; ii) Descripción del proyecto, el cual será estructurado de acuerdo a los lineamientos para la presentación de un artículo científico con el fin de realizar, desde lo académico, un análisis sobre las competencias desarrolladas por los practicantes; el abordaje de la situación problema; metodología; calidad de las propuestas y aceptación de la organización para llevarlas a cabo y fomentar la cultura sobre la participación en eventos académicos para presentar resultados de intervención en las organizaciones y iii) Datos generales de los practicantes, así como correos electrónicos personales y redes sociales que utilicen, a fin de mantenerlos como actores clave, una vez egresados de la licenciatura.

6) Entrega de informes ejecutivos a las organizaciones sobre los resultados del proyecto realizado por los practicantes.

\section{ÁMBITO PROFESIONAL}

Los actores principales en este ámbito son los profesionales de la comunicación en las organizaciones, representados por egresados de escuelas de Comunicación o bien de otras áreas de conocimiento, quienes gestionan la comunicación en las organizaciones. Para conocer sobre ellos, existen estudios sobre trayectorias profesionales que identifican ámbitos de desempeño (Sánchez Olavarría, 2012; 2014), grados de satisfacción sobre las condiciones de trabajo en compañías de medios (García-Borrego, Roses Campos; Farias Batle, 2017), como el Latin American Communication Monitor, que realiza estudio longitudinal sobre la profesión de gestión de comunicación y relaciones públicas en todo el mundo, trabaja con la metodología del European Communication Monitory aplica encuestas a comunicadores de diferentes países latinoamericanos, entre ellos México, y estos estudios han permitido conocer las tendencias sobre el ejercicio profesional. En lo que se refiere ala Universidad de Sonora desde el 2002 realiza estudios de egresados de manera bianual, los estudios son descriptivos y están relacionados con

datos generales que caracterizan a los egresados, su trayectoria educativa y laboral, las condiciones de su empleo actual, la opinión sobre la organización académica e institucional y el plan de estudios, para concluir con una sección sobre la satisfacción con la formación profesional recibida por parte de la Institución. (Universidad de Sonora, 2017)

Sin embargo, son estudios descriptivos que no coadyuvan a la investigación en el subcampo profesional, porque no están presentes las experiencias de los profesionales de la comunicación en las organizaciones que respondan a las preguntas: ¿Qué 
es lo que hace en las organizaciones? ¿Cómo resuelve los problemas de comunicación en la organización? ¿Qué aportaciones realiza para hacer competitiva a la organización? ¿Qué competencias ha desarrollado en su práctica profesional? ¿Cuáles son los principales problemas de comunicación en las organizaciones? ¿Qué tipo de capacitación recibe? ¿Sus intervenciones son estratégicas, tácticas u operativas? ¿Qué lo hace competitivo? ¿Cuáles son los principales obstáculos para el posicionamiento de los comunicadores en las organizaciones? ¿Qué aportaciones haría a los planes de estudio? ¿Qué recomendaciones haría a los estudiantes en su formación profesional? ¿Cuál es su concepto de comunicación organizacional? ¿En qué IES se formó? ¿Cuáles son los determinantes para que los espacios de comunicación sean ocupados por los comunicadores organizacionales? ¿Cómo estructura sus equipos de trabajo? ¿Cuál es el capital humano para atender la comunicación? ¿Qué modelos aplica para el desarrollo de un plan de comunicación? ¿Cómo alinea la comunicación con la misión y visión de la organización? ¿Qué variables e indicadores establece para evaluar la comunicación? ¿Cómo influye la comunicación en la competitividad de la organización? ¿Qué valores hace competitivos a los comunicadores organizacionales? ¿Qué necesidades de comunicación tienen las organizaciones? ¿Cuál es el método, técnicas y sus instrumentos para evaluar la comunicación?

\section{CÓMO IMPLEMENTAR LA ESTRATEGIA}

1) Acercar a las IES, de manera proactiva, a los profesionales de la comunicación que sean egresados de Comunicación.

2) Identificar e incluir en el estudio a responsables de la comunicación, formados en otras áreas del conocimiento, en las organizaciones.

3) Organizar conversatorios, ciclos de conferencias y programas radiofónicos con los profesionales de la comunicación para que expongan sobre sus experiencias. Un escenario más es el SEMCO.

4) Hacer públicas las exposiciones para socializar la profesión. La estrategia posibilitará que los estudiantes, maestros, organizaciones y sociedad en general conozcan sobre el ejercicio profesional del comunicador y la importancia de la comunicación en las organizaciones.

\section{COORDINACIÓN DE LA PROPUESTA}

La propuesta que se presenta tiene viabilidad, dada las experiencias registradas en el ámbito educativo con las prácticas profesionales, la vinculación con las organizaciones y el acercamiento con egresados del Eje Especializante de Comunicación Organizacional de la Universidad de Sonora, a través de conversatorios donde han compartido sus experiencias sobre la práctica profesional que realizan en las organizaciones, de manera espontánea, frente a académicos y estudiantes.

Además se tiene experiencia en el diseño de formato para la recuperación de información sobre el registro y reporte de proyectos de prácticas profesionales de comunicación en las organizaciones, los cuales hasta la fecha siguen vigentes desde su implementación en el ciclo escolar 2007-2.

Para que la propuesta (Figura 3) se sume a los estudios de la Comunicación Organizacional en México, se propone a la AMIPCO la coordinación de los trabajos con base en la siguiente estrategia:

1) Invitar a la red de investigadores y profesionales de la comunicación a una reunión para la presentación de la propuesta metodológica. El SEMCO puede ser un recurso para la socialización de la propuesta. 
2) Para el desarrollo de la propuesta, se requiere de un trabajo colaborativo por lo que se necesita la conformación de grupos de trabajo para el diseño y validación de instrumentos; diseño de base de datos para el registro de la información de acuerdo con el instrumento aplicado en las organizaciones, así como el diseño de un sistema informático para la captura de los proyectos de comunicación que realizarán los practicantes.

3) Los resultados del estudio contribuirán a la elaboración de ponencias, artículos en revistas, capítulos de libro y libros, así como a la consolidación de la red AMIPCO.

4) La AMIPCO, a través de la Red, mantendrá la vinculación permanente con practicantes, colaboradores de la investigación, organizaciones, profesionales de la comunicación y académicos, y su impacto se verá reflejado en el registro de nuevos socios.

5) Con la investigación, se aportaría al Subcampo profesional al sistematizar el estudio de la comunicación en las organizaciones desde la dimensión praxeológica.

6) Identificar las tendencias de la comunicación en México.

7) La estrategia buscará la legitimación del Campo de la Comunicación a través del Subcampo profesional de la Comunicación Organizacional.

8) Con base en los estudios y el diseño de base de datos, generará propuestas relacionadas con la configuración de directorio de organizaciones, egresados y profesionales de la comunicación; la aplicación de instrumentos de autoría, para el estudio cuantitativo y cualitativo de la comunicación en las organizaciones; la realización de estudios comparativos sobre la comunicación organizacional en el ámbito local, regional, nacional e internacional.

Figura 3: Coordinación de la propuesta

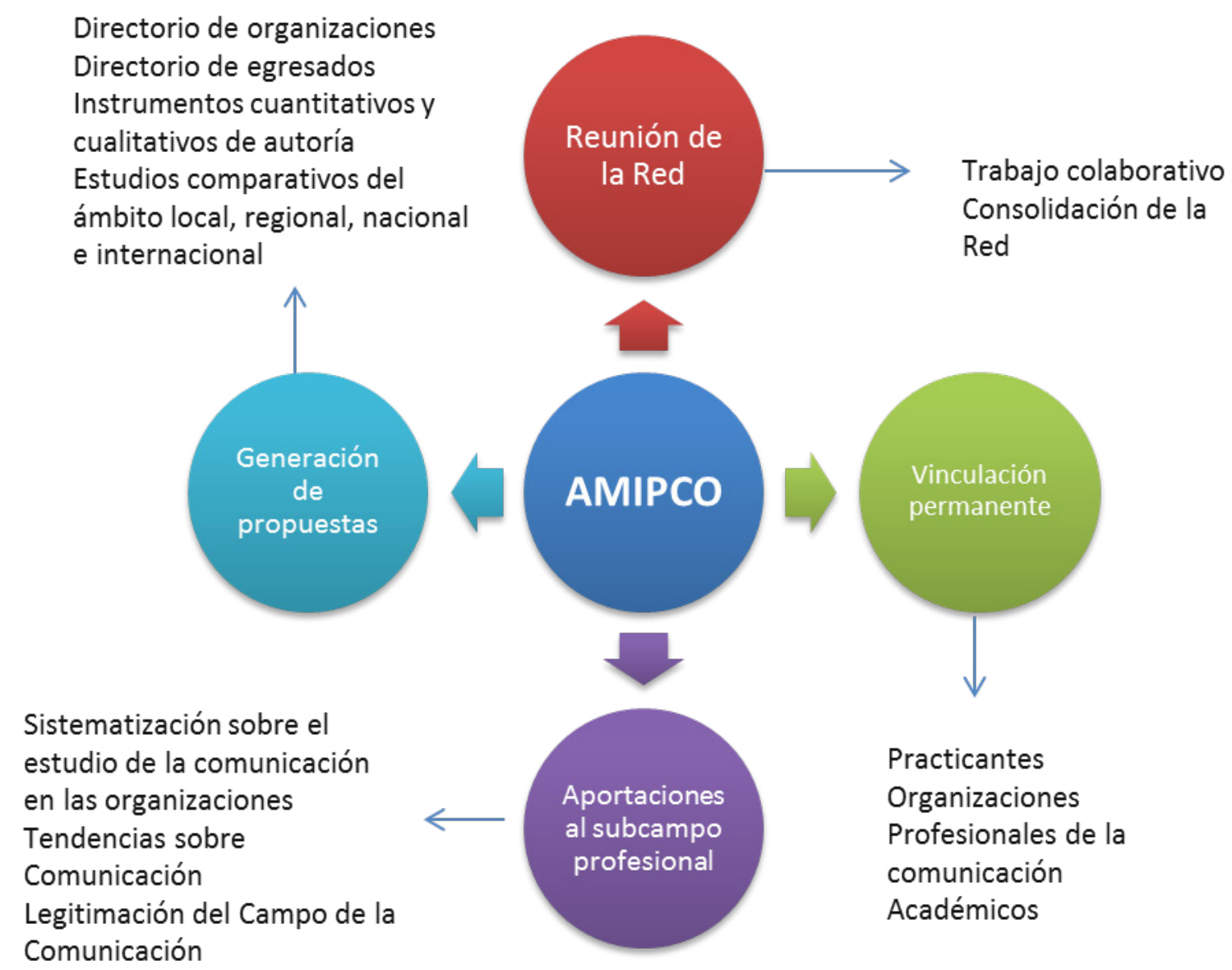

Fuente: Elaborada por los autores. 


\section{CONCLUSIONES}

Es evidente que la propuesta requiere una discusión colegiada, sobre todo si la AMIPCO, a través de sus representantes, considerar pertinente aceptar la coordinación de los trabajos y la viabilidad del proyecto, aunque en un contexto de experiencia positiva a este respecto, es necesario reconocer las limitaciones que presentan las regiones para proponer el desarrollo de la propuesta a corto, medio y largo plazo.

Finalmente, para el cierre de la justificación de la propuesta, la red está conformada por académicos en IES que de alguna manera han tenido relación con practicantes, organizaciones y profesionales de la comunicación, a través de las funciones sustantivas que realizan en docencia, investigación, difusión y divulgación; así como en los procesos de certificación y acreditación que solicitan las Instituciones de Educación Superior y que hacen partícipes a los actores clave en esta propuesta.

\section{REFERENCIAS}

FUENTES NAVARRO, Raúl. La emergencia de un campo académico: continuidad utópica y estructuración científica de la investigación de la comunicación en México. Tlaquepaque: Iteso, 1998

FUENTES NAVARRO, Raúl. Centralidad y marginalidad de la comunicación y su estudio. Guadalajara: Iteso, 2015

GARCÍA-BORREGO, Manuel; ROSES CAMPOS, Sergio; FARIAS BATLE, Pedro. Condiciones de las prácticas profesionales en medios de comunicación: un estudio empírico. Revista Latina de Comunicación Social, Tenerife, n.72, p.430-452, 2017

MATUS VERDUGO, Manuela; VALENZUELA, Blanca; ESTUPIÑÁN MUNGUÍA, Jorge. Prácticas profesionales de comunicación en las organizaciones. In: OJEDA ORTA, María Elizabeth et al (comp.). Las ciencias informáticas: generación del conocimiento en entornos educativos: un enfoque multidisciplinario. México, DF: ILCSA, 2017. p.1162-1117.

SÁNCHEZ OLAVARRÍA, César. La práctica profesional del comunicador de la Universidad del Altiplano: un panorama de su trayectoria. Perfiles Educativos, México, DF, v.34, n.137, p.119-144, 2012.

SÁNCHEZ OLAVARRÍA, César. Los egresados de comunicación y el mercado laboral: un estudio de trayectorias profesionales. Revista Iberoamericana de Educación Superior, México, DF, v.5, n.13, p.40-54, 2014.

CONSUELO Vásquez, profesora de la Universidad de Québec , en Canadá, nos habla sobre la Comunicación Constitutiva de las Organizaciones (CCO), la importancia del trabajo que realiza la \#AMIPCO y de la formación de una red latinoamericana de investigadores de CO. [S.l.:S.n.], 2017. 1 vídeo (5 min). Facebook: Amipco. Disponível em: https://bit.ly/2YOFlCF. Acesso em: 23 jul. 2019.

Unesco. Declaración mundial sobre la educación superior en el siglo XXl: visión y acción. Paris: Unesco, 1998.

UNIVERSIDAD DE SONORA. Convenios de intercambio y colaboración: dirección de vinculación y difusión. Hermosillo: Universidade de Sonora, 2018. Disponível em: https://bit.ly/2ZbDaZG. Acesso em: 23 jul. 2019. 